\title{
Thermal phase transitions for Dicke-type models in the ultrastrong-coupling limit
}

\author{
M. Aparicio Alcalde, ${ }^{1}$ M. Bucher, ${ }^{2}$ C. Emary, ${ }^{2}$ and T. Brandes ${ }^{2}$ \\ ${ }^{1}$ Instituto de Física Teórica, UNESP-São Paulo State University, Caixa Postal 70532-2, 01156-970 São Paulo, SP, Brazil \\ ${ }^{2}$ Institut für Theoretische Physik, Hardenbergstr. 36, TU Berlin, D-10623 Berlin, Germany
}

(Received 12 April 2012; published 18 July 2012)

\begin{abstract}
We consider the Dicke model in the ultrastrong-coupling limit to investigate thermal phase transitions and their precursors at finite particle numbers $N$ for bosonic and fermionic systems. We derive partition functions with degeneracy factors that account for the number of configurations and derive explicit expressions for the Landau free energy. This allows us to discuss the difference between the original Dicke (fermionic) and the bosonic case. We find a crossover between these two cases that shows up, for example, in the specific heat.
\end{abstract}

DOI: 10.1103/PhysRevE.86.012101

PACS number(s): 05.30.Rt, 64.60.an, 32.80.-t, 42.50.Nn

The Dicke model, in its original weak-coupling and multimode form [1] has a long history as a paradigm for collective dissipation [2], and, in its single-mode form, as a test bed for fundamental concepts such as the quantum-classical relation [3,4], scaling [5], or entanglement [9] near quantum phase transitions. Interest in the Dicke superradiance model has been furthered by the recent discovery of the "Hepp-Lieb"-type quantum phase transition $[6,7]$ with Bose-Einstein condensates in an optical cavity [8]. As a mean-field-type phase transition, the full phase diagram in the temperature-coupling constant plane was derived early [10-12] in the thermodynamic limit of $N \rightarrow \infty$ particles (cf. also [13]). In this Brief Report, we re-examine the thermal properties of (a somewhat generalized version of) this model with particular emphasis on the influence of the quantum statistics on the quantum phase transition. We only consider the ultrastrong-coupling limit between the atoms and the light (corresponding to the superradiant phase at low temperatures), but we distinguish between various cases of $N$ bosons or $N$ fermions distributed among $N_{s}$ two-level sites. In particular, we derive simple expressions for the thermodynamic partition sums that can be used to easily calculate thermodynamic quantities such as the specific heat at finite particle number $N$, and to follow an interesting crossover between the case of $N$ bosons on one $\left(N_{s}=1\right)$ site and the original Dicke case of $N$ fermions on $N_{s}=N$ sites.

As a starting point we use the Dicke Hamiltonian with the single bosonic mode $a, a^{\dagger}$ of frequency $\omega$. The angular momentum operators $J_{z}, J^{ \pm}$describe an ensemble of $N$ twolevel atoms with a level splitting $\omega_{0}$. The single-mode Dicke Hamiltonian is

$$
\mathcal{H}=\omega a^{\dagger} a+\omega_{0} J_{z}+\frac{g}{\sqrt{N}}\left(a+a^{\dagger}\right)\left(J^{+}+J^{-}\right) .
$$

A unitary transformation with $U=e^{\sigma J_{z}} e^{i \frac{\pi}{2} J_{y}}$ and $\sigma \equiv$ $\frac{2 g}{\sqrt{N} \omega}\left(a^{\dagger}-a\right)$ rotates and polaron-transforms the Hamiltonian into $\mathcal{H}^{\prime} \equiv U \mathcal{H} U^{\dagger}$ with

$$
\mathcal{H}^{\prime}=-\frac{\omega_{0}}{2}\left(J^{+} e^{\sigma}+J^{-} e^{-\sigma}\right)+\omega a^{\dagger} a-\frac{(2 g)^{2}}{N \omega} J_{z}^{2} .
$$

The Hamiltonian $\mathcal{H}^{\prime}$ can be used as a starting point for a perturbation theory in $\omega_{0}$, that is, around the limit of very large coupling $g \rightarrow \infty$ between the angular momentum and the photon mode. In this limit, the physics is then determined by a (trivial) free photon Hamiltonian $\omega a^{\dagger} a$ and the angular momentum part $\propto J_{z}^{2}$. The analysis of the properties of the thermodynamic partition sum,

$$
Z_{N} \equiv \operatorname{Tr} e^{-\beta \mathcal{H}_{N}}, \quad \mathcal{H}_{N} \equiv-\frac{(2 g)^{2}}{N \omega} J_{z}^{2},
$$

for different physical realizations of $J_{z}$ is the aim of this Brief Report.

The role of particle statistics in $Z_{N}$ can be qualitatively understood by considering the existence or otherwise of a thermal phase transition for $\mathcal{H}_{N}$ in the limit of $N \rightarrow \infty$. To this end, let us first recall that in the original Dicke model, $J_{z}=\frac{1}{2} \sum_{n=1}^{N} \sigma_{n}^{z}$ is the sum of $N$ individual (pseudo)-spin- $\frac{1}{2}$ operators. Superradiant states with maximal spin polarization are then energetically favored by $\mathcal{H}_{N}$, but there are only two configurations (all spins pointing either up or down) where that is achieved. All other spin configurations have larger (nonzero) entropy such that thermal fluctuations trigger a phase transition to a thermally disordered (normal) phase above a critical temperature.

On the other hand, if the state space is restricted to the highly symmetric Dicke states $|J, M\rangle$, the configuration space is much smaller and there is no gain in entropy for states with higher energy. In this situation, which corresponds to $N$ bosons occupying either the upper or lower level of a two-level system, a thermal phase transition does not occur.

The partition function and the number of configurations. We start with discussing the degeneracy factors that appear when evaluating the partition sum $Z_{N}$. Let us assume a configuration space with $N$ particles distributed among $N_{s}$ two-level "sites" $i$, all of which have the same up-level $\uparrow$ and down-level $\downarrow$ energies. We write $J_{z} \equiv \frac{1}{2} \sum_{i=1}^{N_{s}}\left(n_{i \uparrow}-n_{i \downarrow}\right)$ with number operators $n_{i \uparrow}, n_{i \downarrow}$ such that

$$
Z_{N}=\sum_{n=0}^{N} c_{n} e^{\beta \frac{g^{2}}{N \omega}(N-2 n)^{2}},
$$

where $c_{n}$ is the number of configurations with $n$ particles in the down-levels $\downarrow$ and $N-n$ in the up-levels $\uparrow$.

Fermions. (i) For fermions, the number of configurations for $n$ particles in the down-levels is $\left(\begin{array}{c}N_{s} \\ n\end{array}\right)$, and for the remaining $N-n$ particles in the up-levels it is $\left(\begin{array}{c}N_{s} \\ N-n\end{array}\right)$; consequently, $c_{n}=\left(\begin{array}{c}N_{s} \\ n\end{array}\right)\left(\begin{array}{c}N_{s} \\ N-n\end{array}\right)$. (ii) For fermions with no two particles on the same site $i$, one has a restricted choice once all the $\uparrow$ (or the $\downarrow$ ) are occupied and thus $c_{n}=\left(\begin{array}{c}N_{s} \\ n\end{array}\right)\left(\begin{array}{c}N_{s}-n \\ N-n\end{array}\right)$. For the 
particular case $N_{s}=N$, this corresponds to the $N$ localized and distinguishable (pseudo)spins in the original Dicke model discussed above and gives the partition sum,

$$
Z_{N}^{\mathrm{D}} \equiv \sum_{n=0}^{N}\left(\begin{array}{l}
N \\
n
\end{array}\right) e^{\beta \frac{g^{2}}{N \omega}(N-2 n)^{2}},
$$

which corresponds to the partition sum of the Lipkin-MeskovGlick model at zero magnetic field [14].

Bosons. For $N$ bosons in $m$ single particle levels, there are $\left(\begin{array}{c}N+m-1 \\ m-1\end{array}\right)$ configurations which in our case means

$$
c_{n} \equiv\left(\begin{array}{c}
n+N_{s}-1 \\
N_{s}-1
\end{array}\right)\left(\begin{array}{c}
N-n+N_{s}-1 \\
N_{s}-1
\end{array}\right) .
$$

The particular case of a single site $N_{s}=1$ yields $c_{n}=1$ and thus

$$
Z_{N}^{\text {bos }} \equiv \sum_{n=0}^{N} e^{\beta \frac{g^{2}}{N \omega}(N-2 n)^{2}}, \quad N_{s}=1 .
$$

We also obtain this result by representing $J_{z}=\frac{1}{2}\left(2 b^{\dagger} b-N\right)$ via one Holstein-Primakoff boson $b^{\dagger}$, or, alternatively, by writing $J_{z}=\frac{1}{2}\left(b_{\uparrow}^{\dagger} b_{\uparrow}-b_{\downarrow}^{\dagger} b_{\downarrow}\right)$ with two Schwinger boson modes for $\uparrow$ and $\downarrow$ by using $b_{\uparrow}^{\dagger} b_{\uparrow}+b_{\downarrow}^{\dagger} b_{\downarrow}=N$ and noticing that the number $n$ of $\downarrow$ bosons uniquely fixes a configuration which means $c_{n}=1$.

Landau free energy function. The most transparent way to discuss the difference between the two cases Eq. (5) and Eq. (7) and generalizations thereof is by transforming the partition sums into integrals over an order parameter $y$. As we are dealing with a zero-dimensional field theory here, this is particularly simple and is formally achieved by a HubbardStratonovich transformation which here is simply given by the Gaussian integral identity, $e^{x^{2}}=\frac{1}{\sqrt{\pi}} \int_{-\infty}^{\infty} d y e^{-y^{2}+2 x y}$, applied to the respective Boltzmann factors in $Z_{N}$. For the distinguishable (Dicke) case, we use the binomial formula as $\sum_{n=0}^{N}\left(\begin{array}{l}N \\ n\end{array}\right) e^{z(N-2 n)}=(2 \cosh z)^{N}$ to obtain (after substituting $y \rightarrow \sqrt{N} y)$

$$
\begin{aligned}
Z_{N}^{\mathrm{D}} & =\sqrt{\frac{N}{\pi}} \int_{-\infty}^{\infty} d y e^{-N \Phi^{\mathrm{D}}(y)}, \\
\Phi^{\mathrm{D}}(y) & \equiv y^{2}-\ln (2 \cosh (2 \alpha y)), \quad \alpha \equiv g \sqrt{\frac{\beta}{\omega}},
\end{aligned}
$$

where we introduced the dimensionless coupling parameter $\alpha$.

Similarly, for the $N_{s}=1$ boson case, we carry out the geometric progression $\sum_{n=0}^{N} e^{z(N-2 n)}=e^{-N z}\left(e^{2 z(N+1)}-\right.$ 1) $/\left(e^{2 z}-1\right)$ to obtain

$$
\begin{aligned}
Z_{N}^{\text {boson }} & =\sqrt{\frac{N}{\pi}} \int_{-\infty}^{\infty} d y e^{-N \Phi^{\text {boson }}(y)}, \\
\Phi_{N}^{\text {boson }}(y) & \equiv y^{2}+2 \alpha y-\frac{1}{N} \ln \frac{e^{4 \alpha(N+1) y}-1}{e^{4 \alpha y}-1} \quad N_{s}=1 .
\end{aligned}
$$

The so-defined Landau free energy functions $\Phi(y)$ now allow us to elucidate the critical properties of the models.

First, we observe that in the (Dicke) case of distinguishable particles, $\Phi^{\mathrm{D}}(y)$ in Eq. (8) is $N$ independent, and $\Phi^{\mathrm{D}}(y) \equiv \beta f$ is determined by the $\omega_{0}=0$ limit of the usual mean-field
$(N \rightarrow \infty)$ expression for the free energy $f$ per particle [10-12]. As expected, the term $\ln [2 \cosh (2 \alpha y)]$ therefore is the mean-field free energy of a single (pseudo)spin in the fluctuating field $y$. The parameter $\alpha$ determines the shape of $\Phi^{\mathrm{D}}(y)$ and, in the $N \rightarrow \infty$ limit, the position $y_{\mathrm{D}}$ of the minimum of $\Phi^{\mathrm{D}}(y)$ relevant for the asymptotic expression of the integral according to the Laplace method, as given by the self-consistent equation,

$$
y_{\mathrm{D}}=\alpha \tanh 2 \alpha y_{\mathrm{D}} .
$$

This has the unique solution $y_{\mathrm{D}}=0$ when $2 \alpha^{2}<1$ corresponding to temperatures $T>T_{c} \equiv 2 g^{2} / \omega$ larger than the critical temperature $T_{c}$. This solution describes the normal phase whereas for $T<T_{c}$ there exist two minima in $\Phi^{\mathrm{D}}(y)$ that describe the symmetry-broken superradiant phase.

This is in contrast to the bosonic case, where the Landau free energy $\Phi_{N}^{\text {boson }}(y)$ in Eq. (9) is $N$ dependent but acquires a simple form in the thermodynamic limit $N \rightarrow \infty$,

$$
\Phi_{\infty}^{\text {boson }}(y)=y^{2}-2 \alpha|y|,
$$

which has two unique minima at $y= \pm \alpha$ regardless of the value of $\alpha$. This means that in the bosonic case, one is always in the superradiant, symmetry-broken phase and no thermal phase transition into a normal phase occurs.

Thermodynamic behavior. In the following, we discuss the specific heat $C \equiv \beta^{2} \partial_{\beta}^{2} \log \left(Z_{N}\right)$ for both cases (we set $k_{B}=1$ ). In the Dicke case [spin- $\frac{1}{2} \mathrm{~s}$ corresponding to the fermionic partition sum Eq. (5)], Fig. 1, the specific heat per particle can be calculated from $\Phi^{\mathrm{D}}(y) \equiv \beta f$ in the thermodynamic limit $N \rightarrow \infty$ by eliminating derivatives of $y_{D}$ and solving the self-consistent Eq. (10), leading to the expected singular behavior at the transition point $\alpha^{*}=1 / \sqrt{2}$. A simple $1 / N$ expansion of $Z_{N}^{\mathrm{D}}$, Eq. (8), works only well not too close to the critical point, as we have checked, whereas the asymptotics in $N$ at $\alpha^{*}$ is more complicated as has been discussed in [14] recently. Numerically, for finite $N$ the peak height of $C / N$ and the shift of the peak from $\alpha^{*}$ give, however, a very good agreement with a $1 / \sqrt{N}$ correction fit.

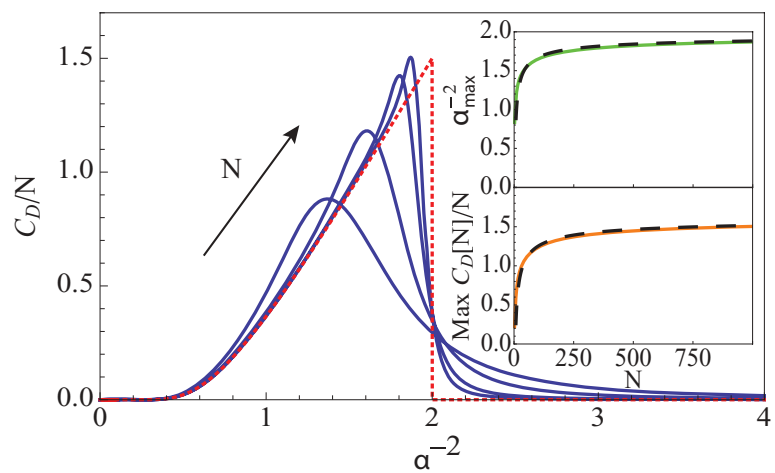

FIG. 1. (Color online) The specific heat per particle $\left(k_{B}=1\right)$ as a function of $\alpha^{-2} \equiv \omega /\left(g^{2} \beta\right)$ (proportional to the temperature) in the Dicke case of $N$ localized spin- $\frac{1}{2} \mathrm{~s}$, [derived from the fermionic partition sum Eq. (5)], for $N=20,75,400$, and 1000 particles (dotted line, $N \rightarrow \infty$ ). (Upper inset) Shift of peak from critical point $\alpha^{*}=$ $1 / \sqrt{2}$ for all $N \leqslant 1000$. (Lower inset) Peak height at maximum. (Dashed lines) Fit of the numerical data with $f_{\text {upper }}^{1} \equiv 1.64-\frac{3.9}{\sqrt{N}}$ and $f_{\text {lower }}^{1} \equiv 1.98-\frac{3.1}{\sqrt{N}}$. 


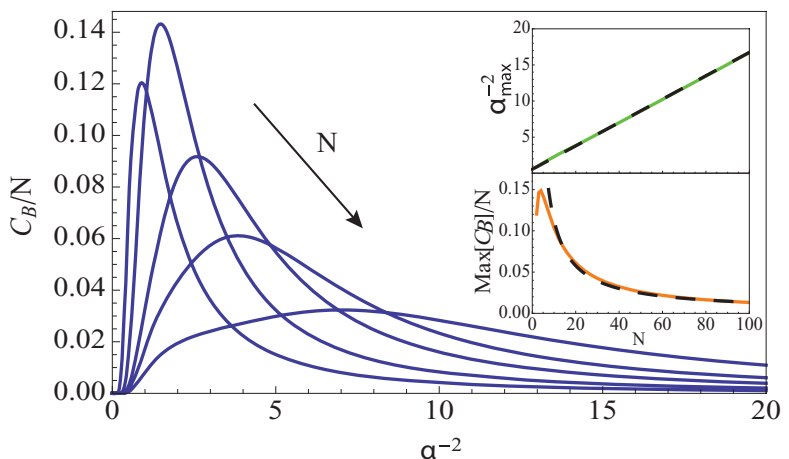

FIG. 2. (Color online) The specific heat per particle as in Fig. 1 but for the bosonic partition sum Eq. (7), for $N=2,5,12,20$, and 40 particles. (Dashed lines) Linear fit $f_{\text {upper }}^{2} \equiv 0.6+0.2 N$ for maximum position (upper inset); $f_{\text {lower }}^{2} \equiv \frac{1.1}{N}$ fit for maximum (lower inset).

In the bosonic case (Fig. 2), the specific heat per particle shows a totally different behavior. For small numbers of bosons $(N=2,3,4), C / N$ first increases with $N$ and then (for $N>4$ ) decreases with $\frac{1}{N}$, as does its maximum. The position $\alpha^{-2}$ of the maximum of $C / N$ is linear for the particle number $N$. In the limit $N \rightarrow \infty$ the specific heat per particle is zero which can be understood from Eq. (11) by using the above-mentioned thermodynamic relations and considering that $y= \pm \alpha$.

Crossover between bosonic and Dicke case. We now discuss an interesting crossover between the two cases obtained above by regarding the bosonic case with $N_{s}>1$. For $N_{s}$ of the order of the particle number $N$, we expect the bosons to spread over many energetically equivalent configurations which, due to the entropy argument given in the introduction, should re-establish the thermal phase transition found in the Dicke (fermionic spin- $\frac{1}{2}$ ) case. We therefore generalize the Landau free energy for the bosonic case Eq. (9) to arbitrary $N_{s}$,

$$
\Phi_{N, N_{S}}^{\text {boson }}(y) \equiv y^{2}-\frac{1}{N} \ln \sum_{n=0}^{N} c_{n} e^{2 \alpha y(N-2 n)},
$$

with $c_{n}$ given by Eq. (6). Figure 3 shows the crossover in the free energy when passing from the bosonic case with $N_{s}=1$ (showing no phase transition when varying $\alpha$ ) to larger degeneracies, where for $N_{s} \gg N$, the free energy $\Phi_{N, N_{S}}^{\text {boson }}(y)$ becomes equivalent to the one of the Dicke case Eq. (8). This can be understood by using Stirling's formula to expand the number of configurations $c_{n}$ [Eq. (6)], which leads to $c_{n} \sim\left(\begin{array}{l}N \\ n\end{array}\right)\left(N_{s} / N\right)^{N}$ and therefore the partition sum,

$$
Z_{N}^{\text {bos }} \sim\left(\frac{N_{s}}{N}\right)^{N} Z_{N}^{\mathrm{D}}, \quad N_{s} \gg N \gg 1 .
$$

Correspondingly, in this limit the bosonic free energy per particle differs from the Dicke free energy by $-k_{B} T \ln \left(N_{s} / N\right)$ which just describes an additional entropy term caused by the enhanced "volume" of configurations. Thus for $N_{s} \gg N$, multiply occupied sites play no role any longer, nor does the statistics of the particles (fermions or bosons), and the only remaining relevant statistical information is the number of occupied upper and lower levels as in the Dicke case. As the entropy gain $k_{B} \ln \left(N_{s} / N\right)$ is just a constant, the specific heat $C / N$ then has to coincide with the specific heat in the Dicke case.

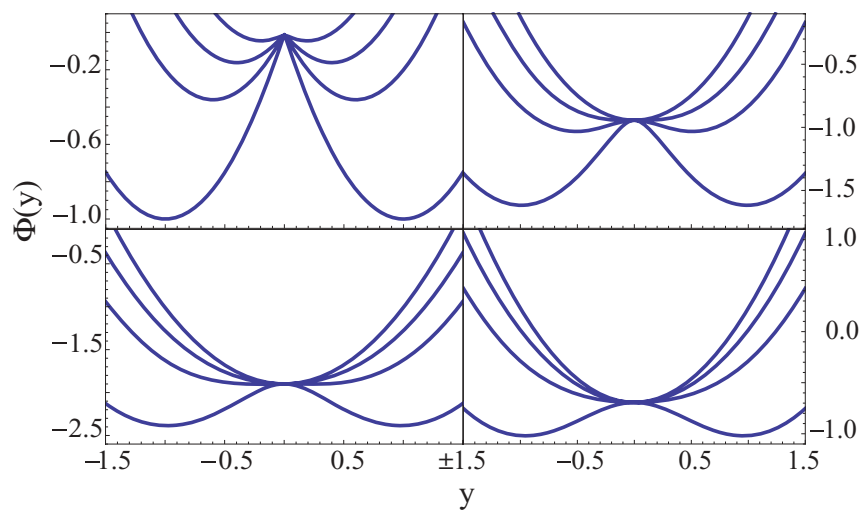

FIG. 3. (Color online) Crossover between bosonic and Dicke case in the Landau free energy, Eq. (12), for $N=400$ bosons and coupling strengths $\alpha=0.2,0.4,0.6,1.0$ within each graph. (Upper left) $N_{s}=1$ $\left(N_{s}\right.$, number of two-level sites); this corresponds to the simple bosonic case Eq. (9) [and Eq. (11) for $N \rightarrow \infty$ ], that is, the absence of a phase transition, since the number of minima remains two irrespective of $\alpha$. The phase transition is re-established for macroscopic degeneracy (upper right, $N_{s}=N / 4$; lower left, $N_{s}=N$ ), since a transition from a double- to single-minimum occurs. The lower right shows the Dicke case $\Phi^{\mathrm{D}}(y)$, Eq. (8), with the phase transition occurring at $\alpha^{*}=1 / \sqrt{2} \approx 0.71$.

This behavior of the specific heat $C / N$ is shown in Fig. 4, where we vary $N_{s}$ from the bosonic case $N_{s}=1$ over $N_{s}=N$ to finally higher values of $N_{s} \gg N$ corresponding to the Dicke (fermionic) form of $C / N$ from Fig. 1. With $N$ fixed, the $C / N$ curves have peaks that shift from larger to smaller values of $\alpha^{-2}$ with increasing $N_{s} / N$ corresponding to a decreasing $N / N_{s}$ in the $N_{s}=1$ bosonic case in Fig. 2. At even larger $N_{s} / N \gg 1$ the curves approach the Dicke form at the critical point $\alpha^{*}$ of the Dicke thermal phase transition. Note that the peaks approach $\alpha^{*}$ from the right here, whereas in the Dicke case (Fig. 1) $N_{s}=N$ was fixed and the $C / N$ peaks approached $\alpha^{*}$ from smaller $\alpha^{-2}$ with increasing $N$.

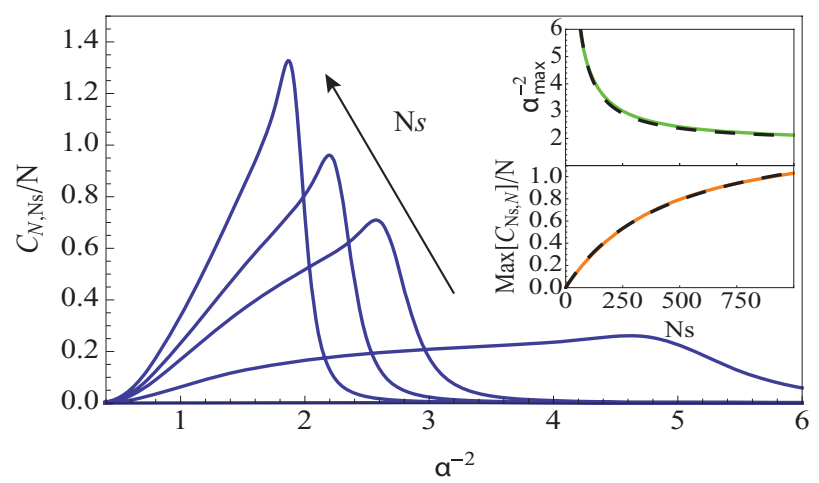

FIG. 4. (Color online) The specific heat per particle at fixed particle number $N=400$ for different number of two-level sites $N_{s}=1$ (bosonic case, almost coincides with $x$ axis), $N_{s}=100$, $N_{s}=400$ (Dicke case), and $N_{s}=600,5000$ as obtained from Eqs. (4) and (6). (Upper inset) With increasing $N_{s}$ the peak position in $C / N$ shifts towards the critical point $\alpha^{-2}=2$ of the Dicke case. (Lower inset) Increase of maximum of $C / N$ with $N_{s}$. (Dashed lines) Fit of the numerical data with $f_{\text {upper }}^{4} \equiv 1.82-\frac{275}{N_{s}}$ and $f_{\text {lower }}^{4} \equiv \frac{1.5 N_{s}}{458.6+N_{s}}$. 
Conclusion and outlook. Our results indicate that the Dicke model displays an interesting thermodynamic behavior if one considers the possible configurations of $N$ particles in $N_{s}$ two-level systems. In the ultrastrong-coupling limit, the model reduces to an atomic self-interaction term for which we have derived explicit expressions for the partition function, Landau free energy, and specific heat. We find a crossover in the specific heat from a bosonic form at small $N_{s}$ (where no thermal phase transition occurs) to the form following from the (original) Dicke model for $N_{s} \gg N \gg 1$ that displays a thermal phase transition at $\alpha^{-2} \equiv k_{B} T \omega / g^{2}=2$ between the normal and the superradiant phase.

In our calculations, we regarded $\mathcal{H}_{N}$ in Eq. (3) as an effective Hamiltonian for the ultrastrong-coupling regime between atoms and light of the Dicke model Eq. (1). In analogy with the simulation of the Dicke model with Bose-Einstein condensates [8], we suggest the various limits of $\mathcal{H}_{N}$ to be regarded as effective models to be simulated with, for example, cold atoms. A further challenge would be an extension to Dicke-type models with finite coupling strengths, multiple levels [15], and degeneracies $N_{s}$ in the bosonic case.

Acknowledgments. We acknowledge support from the DFG through Grant No. SFB 910. M.A.A. thanks M. Hayn, V. Bastidas, B. Pimentel, and N. Svaiter for useful discussions, and the Institut für Theoretische Physik of the TU Berlin for their kind hospitality. M.A.A. acknowledges the State of São Paulo Research Foundation (FAPESP) for financial support.
[1] R. H. Dicke, Phys. Rev. 93, 99 (1954).

[2] T. Brandes, Phys. Rep. 408, 315 (2005).

[3] C. Emary and T. Brandes, Phys. Rev. Lett. 90, 044101 (2003); Phys. Rev. E 67, 066203 (2003).

[4] A. Altland and F. Haake, Phys. Rev. Lett. 108, 073601 (2012); New J. Phys. 14, 073011 (2012).

[5] J. Vidal and S. Dusuel, Europhys. Lett. 74, 817 (2006).

[6] K. Hepp and E. Lieb, Ann. Phys. 76, 360 (1973).

[7] F. Dimer, B. Estienne, A. S. Parkins, and H. J. Carmichael, Phys. Rev. A 75, 013804 (2007).

[8] K. Baumann, C. Guerlin, F. Brennecke, and T. Esslinger, Nature (London) 464, 1301 (2010).
[9] N. Lambert, C. Emary, and T. Brandes, Phys. Rev. Lett. 92, 073602 (2004).

[10] Y. K. Wang and F. T. Hioe, Phys. Rev. A 7, 831 (1973).

[11] K. Hepp and E. Lieb, Phys. Rev. A 8, 2517 (1973).

[12] H. J. Carmichael, C. W. Gardiner, and D. F. Walls, Phys. Lett. A 46, 47 (1973).

[13] M. Aparicio Alcalde, A. L. L. de Lemos, and N. F. Svaiter, J. Phys. A 40, 11961 (2007); M. Aparicio Alcalde and B. M. Pimentel, Physica A 390, 3385 (2011).

[14] J. Wilms, J. Vidal, F. Verstraete, and S. Dusuel, J. Stat. Mech. (2012) P01023.

[15] M. Hayn, C. Emary, and T. Brandes, Phys. Rev. A 84, 053856 (2011). 